This item was submitted to Loughborough's Institutional Repository (https://dspace.lboro.ac.uk/) by the author and is made available under the following Creative Commons Licence conditions.

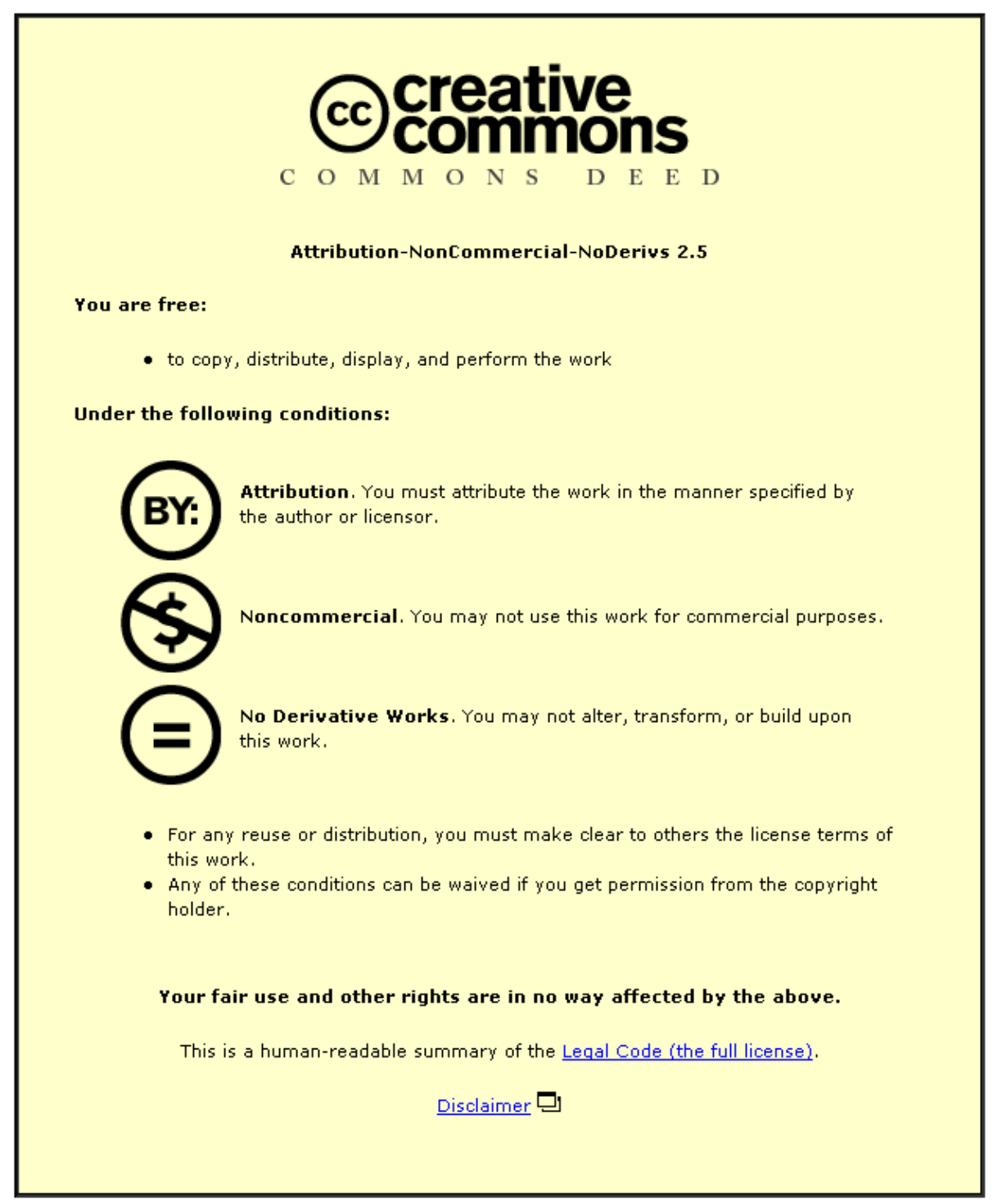

For the full text of this licence, please go to: http://creativecommons.org/licenses/by-nc-nd/2.5/ 


\title{
Evaluation of impact sound of the 'feel' of a golf shot
}

\author{
J.R. Roberts ${ }^{\mathrm{a}}$, R. Jones ${ }^{\mathrm{a}}$, N.J. Mansfield ${ }^{\mathrm{b}}$, S.J. Rothberg ${ }^{\mathrm{a}}$ \\ ${ }^{a}$ Wolfson School of Mechanical and Manufacturing Engineering, Loughborough University, Loughborough, \\ Leicestershire, LE11 3TU, UK \\ ${ }^{\mathrm{b}}$ Department of Human Sciences, Loughborough University, Loughborough, Leicestershire, LE11 3TU, UK
}

\begin{abstract}
The 'feel' of a golf club is an important characteristic that has a significant influence on a golfer's choice of equipment. The sound from impact varies between different clubs and balls and this has been found to contribute to the feel of a shot. The aim of this study was to investigate the relationship between the impact sound and elite golfers' subjective perceptions of the shot. Suitable test procedures were developed to quantify the golfers' perceptions using a questionnaire and to measure the impact sound from the same shots. Statistical techniques were then employed to identify correlations between parameters of the impact sound and the golfers' subjective ratings. The characteristics sharpness and loudness of sound and pleasantness and liveliness of feel were found to be strongly correlated; a shot was rated as having a pleasant feel if it had a loud, sharp sound and a lively feel. Strong positive correlations were also obtained between the subjective ratings and parameters of the impact sound such as sound pressure level, loudness level (according to ISO 532) and sharpness.
\end{abstract}

\section{Introduction}

The sound of a golf impact has been found to contribute significantly to a golfer's perception of a shot [1-5]. Variations in sound characteristics between different clubs, such as the pitch, tone colour, loudness and duration of the impact sounds, are detected by the golfers and affect the desirability of a club. Sound also appears to have a psychological effect on the golfer; louder, crisper sounds, generated by modern, hollow metal clubheads, give the golfer the impression that the ball has been propelled from the clubface faster and will, therefore, travel further. It may also be possible that some of the feel characteristics that have previously been thought to relate to tactile sensations at impact [6] are influenced more by the impact sound. These findings arose from the analysis of golfers' responses obtained during open-ended interviews, which involved elite players hitting shots with a selection of modern and traditional drivers [1].

To develop these concepts further, controlled tests were performed in which relationships between the golfers' subjective perceptions of the feel of a shot, quantified using a questionnaire, and the characteristics of the measured impact sound were investigated. The objective of this work is to develop a greater understanding of the way in which golfers' perceive the sound from impact. If this can be achieved then it may eventually be possible to predict the feel of a club due to its impact sound using modelling techniques (e.g. finite element analysis) and the sound characteristics of a clubhead could be manipulated earlier in the design process. This paper reports the first stage of this process, in which the development of a suitable test methodology and data analysis procedure is of as much importance as familiarisation with the nature of the impact sounds and the identification of correlations between golfers' perceptions and objective data.

\section{Characteristics of golf club impact sound}

Despite the acknowledged importance of impact sound on players' perceptions [2-10], only a few studies of the sound characteristics of sports equipment have actually been undertaken and all have been related to golf. Wicks et al. [2] compared the dynamic properties of forged and cast clubheads but found little difference in the natural frequencies of the whole clubs or the acoustic response of the heads. Hocknell et al. [3] compared sound and vibration measurements from shots hit using a golf robot with an averaged opinion of the feel of different club-ball combinations constructed from golfers' perceptions. Frequencies in the range $5-11 \mathrm{kHz}$ were found to dominate the sound from a hollow metal driver, much higher than the frequency content for a traditional wooden driver, which did not exceed $3.5 \mathrm{kHz}$. The golfers generally preferred the lower pitched sounds from the wooden clubhead although a considerable percentage found the higher pitched sounds from the metal head to be acceptable. More recent studies, however, have found that few golfers prefer the feel of the traditional clubheads $[1,11]$, which suggests a change in golfers' opinions over the last five years as players have become more accustomed to the feel of modern equipment offering superior performance. In a separate but related study [4], Hocknell et al. conducted a modal analysis of a clubhead 
and identified correlations between the natural frequencies of the head and peaks in the impact sound spectra. The largest peaks in the sound spectra were found to correspond with vibration mode shapes that involved more than one surface of the clubhead.

Kuwano et al. [5] asked ten subjects to rate the sounds from numerous different drivers using seven-point adjective scales ranging from "hard-soft", "sharp-dull", "refreshing-not refreshing", "powerful-weak" and "vividdead". Strong correlations were obtained between the subjects' perceptions and the psychoacoustic metrics loudness and sharpness of the measured impact sounds. The authors also investigated time variations in sharpness and found that the difference in sharpness from the point of hitting to $60 \mathrm{~ms}$ later correlated strongly with subjective perceptions. In particular, an increase in sharpness over this period was perceived to be more refreshing and vice versa.

There are strong similarities between this study and the testing conducted by Kuwano et al. The significant difference is that Kuwano et al. collected subjective data from jury trials where golf impact sounds were presented to subjects through headphones. In this study, however, perceptions were obtained directly from elite golfers hitting shots with a range of clubs under real play conditions.

\section{Human Response to impulsive sounds}

Impact sounds in golf are short in duration and, from this study, it has been found that they contain a broad range of frequencies (mainly between 1 and $10 \mathrm{kHz}$ ), whilst the instantaneous sound pressure level, measured at the ear, can reach peaks of up to $127 \mathrm{~dB}$. Impulsive sounds such as these will have certain perceived qualities, such as loudness, pitch, timbre, duration, sharpness, volume and density that can be linked to qualities of the acoustic pressure and its frequency content, although the relationships are often complex.

Perceived loudness is generally related to A-weighted sound pressure level (SPL(A)). This metric is only suitable, however, for approximating the perceived loudness of pure tones or narrow band noises at lower levels, as SPL(A) does not fully account for the effect of level, bandwidth, frequency content and duration on the perceived loudness of a sound [12].

Zwicker's psychoacoustic model calculates loudness level by transforming a sound spectrum into a specific loudness distribution, the area below which is proportional to the perceived loudness of the sound [13]. Zwicker's model is widely employed in sound quality evaluations and was also used in this study despite being developed for steady, continuous sounds. Previous studies have found that, for impulsive sounds shorter than 100$200 \mathrm{~ms}$, the loudness reduces progressively as the duration of the sound is decreased [14-17]. Studies indicate that below approximately $200 \mathrm{~ms}$, an increase of $3 \mathrm{~dB}$ in sound pressure level is necessary to maintain the same loudness when the pulse duration is halved $[16,17]$.
Zwicker's original model, however, was not designed to take into account this effect of sound duration on perceived loudness.

As for loudness, the ear requires a tone to be of a minimum duration to detect pitch accurately. For tones shorter than a critical duration, the auditory system has insufficient time to determine a stable, definite pitch and the tone will be heard as a click, regardless of frequency. Gulick concluded that for tones above $1 \mathrm{kHz}$, the critical duration is a fixed length of time, $10 \mathrm{~ms}$ [17]. Even for tones that exceed the minimum duration, the tonal quality continues to improve as duration is increased to about $250 \mathrm{~ms}$, above which further increases do not result in improved discrimination $[17,18]$. Tones of duration between 10 and $250 \mathrm{~ms}$ have a tonal quality described as 'click-pitch' [19].

A golf impact sound is dominated by frequencies above $1 \mathrm{kHz}$ and its duration can range from a few milliseconds with heavily damped clubheads to tens of milliseconds with lightly damped, hollow, metal clubheads and. This is shorter than the $\mathbf{2 5 0} \mathrm{ms}$ required by the auditory system to detect sound characteristics accurately and, therefore, the variations in sound duration between clubs are likely to have an effect on the golfers' perceptions of both pitch and loudness. The duration of the impact sounds was difficult to quantify so, to compare differences in duration between clubs, a method of calculating the decay rate of the sound envelope was developed and is described in more detail later in the paper.

The pitch strength of a sound is dependent on its frequency content as well as its duration and sounds with continuous spectra, such as golf impact sounds, generally have weak pitch strengths. As a result, golfers' perceptions may be more influenced by the tone colour of a sound and, in particular, its sharpness - a distinct sensation that contributes to tone colour. Von Bismark defined equations for calculating the sharpness of a sound, which is given by the weighted first moment of the specific loudness distribution [20]. This method has since been found to be too level dependent and a modification has been proposed by Aures [21].

In this study, both traditional metrics, such as sound pressure level, and psychophysical measures, such as loudness and sharpness, are correlated with the golfers' perceptions to investigate the relationships that exist.

\section{Test methodology}

Players' perceptions of different drivers and ball types were obtained during interviews with elite golfers as part of a previous study [1]. From the analysis of the golfers' responses, ten general dimensions related to the feel of a golf shot emerged along with the vocabulary used by the players. Feel characteristics thought to be related to the sound of impact were selected for this study.

During the interviews, the golfers frequently used terms such as 'dead', 'dull', 'explosive', 'tinny', 'crisp' and 'sharp' to describe their perception of the impact sound. These descriptors suggest that the golfers perceived differences 
in the loudness and sharpness of the sounds and so these two characteristics were selected for investigation in this study.

Golfers also described their perception of the interaction between the ball and the clubhead during impact. With the traditional, wooden headed clubs they felt the ball being 'absorbed' by the clubhead, apparently increasing the duration of impact and, in the players' view, decreasing the speed at which the ball left the clubhead; this was often described as a 'dead' or 'dull' feel. In contrast, with modern, metal clubheads, they felt that the ball did not compress as much and then would 'shoot' off the clubhead, apparently reducing the impact duration and, in their view, increasing the post impact ball speed. A previous study [11] discovered little correlation between the golfers' descriptions and measured impact durations with different head constructions and ball types, and it was postulated that their perceptions had been influenced by the impact sound. The perceived speed of the ball off the clubface was, therefore, another characteristic selected for inclusion in this study.

The terms 'hard' and 'soft' were other popular descriptors used by the golfers to describe the feel from impact. The hardness of feel was associated with the type of clubhead, the ball construction and also the impact location on the clubface. Although a number of the golfers linked the hardness of feel to tactile sensations, it was suspected that impact sound may also contribute and so hardness was chosen as another feel characteristic to investigate. The overall pleasantness of feel was selected as the final perception to measure.

\subsection{Measurement of subjective data}

The use of psychometric instruments to measure players' opinions of sports equipment has been rare and, in the few cases where techniques have been reported, the methods used have varied in quality. Techniques that have previously been employed include using a category scale [9] or a visual analogue scale [10], providing a choice of alternative responses [22] and obtaining rankings [3].

For the purposes of this study, scaled response questions were identified as an appropriate method for quantifying the golfers' perceptions. For each feel characteristic selected, a question was phrased and the golfer given a choice of responses from 1 to 9 with descriptive words used to give the scale orientation. The five questions used are listed in Table 1 in the same format as presented to the players. Kuwano et al used similar descriptive words in their study of golf impact sounds [5].

A problem with this question format is that, initially, participants will be unfamiliar with the style of the questions and will take some time to develop their own reference levels. The results from the first club were, therefore, removed from the analysis and the club order randomised to minimise order effects.

\subsection{Measurement of objective data}

Impact sound was measured using a microphone situated at a horizontal distance of $90 \pm 10 \mathrm{~cm}$ from the ball and at a height of $140 \pm 10 \mathrm{~cm}$, i.e. at the approximate location of the head of a left-handed golfer addressing the same ball, and was directed at the golf ball in accordance with IEC recommendations, as illustrated in Figure 1. A windshield was used on the microphone. The unweighted measurement was collected using a computer based data acquisition system; each signal was sampled at 51,200 samples per second for $80 \mathrm{~ms}$ with a $20 \mathrm{kHz}$ low pass filter used to prevent aliasing.

\subsection{Test procedure}

Twenty elite golfers (handicaps less than 5), aged between 20 and 55, participated in the tests but data from three golfers, Subjects 3, 9 and 12, were later rejected because either their ball striking was inconsistent or they displayed an obvious lack of concentration during the test. The remaining seventeen golfers had a mean age of 35 years, $\sigma=8.5$ years. One of the golfers was a Tour professional, eight were club professionals, six were assistant professionals and two were amateur golfers. Nine of the golfers were American of which eight were male and one was female, the remaining eight were

Table 1. Questions used to quantify golfers' perceptions of five feel characteristics

\begin{tabular}{|c|c|c|c|c|c|c|c|c|c|c|}
\hline \multicolumn{11}{|c|}{ How did the shot feel? } \\
\hline Unpleasant & 1 & 2 & 3 & 4 & 5 & 6 & 7 & 8 & 9 & Pleasant \\
\hline \multicolumn{11}{|c|}{ How did the impact sound? } \\
\hline Very dull & 1 & 2 & 3 & 4 & 5 & 6 & 7 & 8 & 9 & Very crisp, sharp \\
\hline \multicolumn{11}{|c|}{ How loud was the impact sound? } \\
\hline $\begin{array}{l}\text { Quiet, dead } \\
\text { sound }\end{array}$ & 1 & 2 & 3 & 4 & 5 & 6 & 7 & 8 & 9 & $\begin{array}{l}\text { Loud, explosive } \\
\text { sound }\end{array}$ \\
\hline \multicolumn{11}{|c|}{ How did the impact feel in your hands? } \\
\hline Very soft & 1 & 2 & 3 & 4 & 5 & 6 & 7 & 8 & 9 & Very hard \\
\hline \multicolumn{11}{|c|}{ How quickly did you perceive the ball to have come off the clubface? } \\
\hline $\begin{array}{l}\text { Dead, ball came } \\
\text { off slowly }\end{array}$ & 1 & 2 & 3 & 4 & 5 & 6 & 7 & 8 & 9 & $\begin{array}{l}\text { Lively, ball came } \\
\text { off quickly }\end{array}$ \\
\hline
\end{tabular}




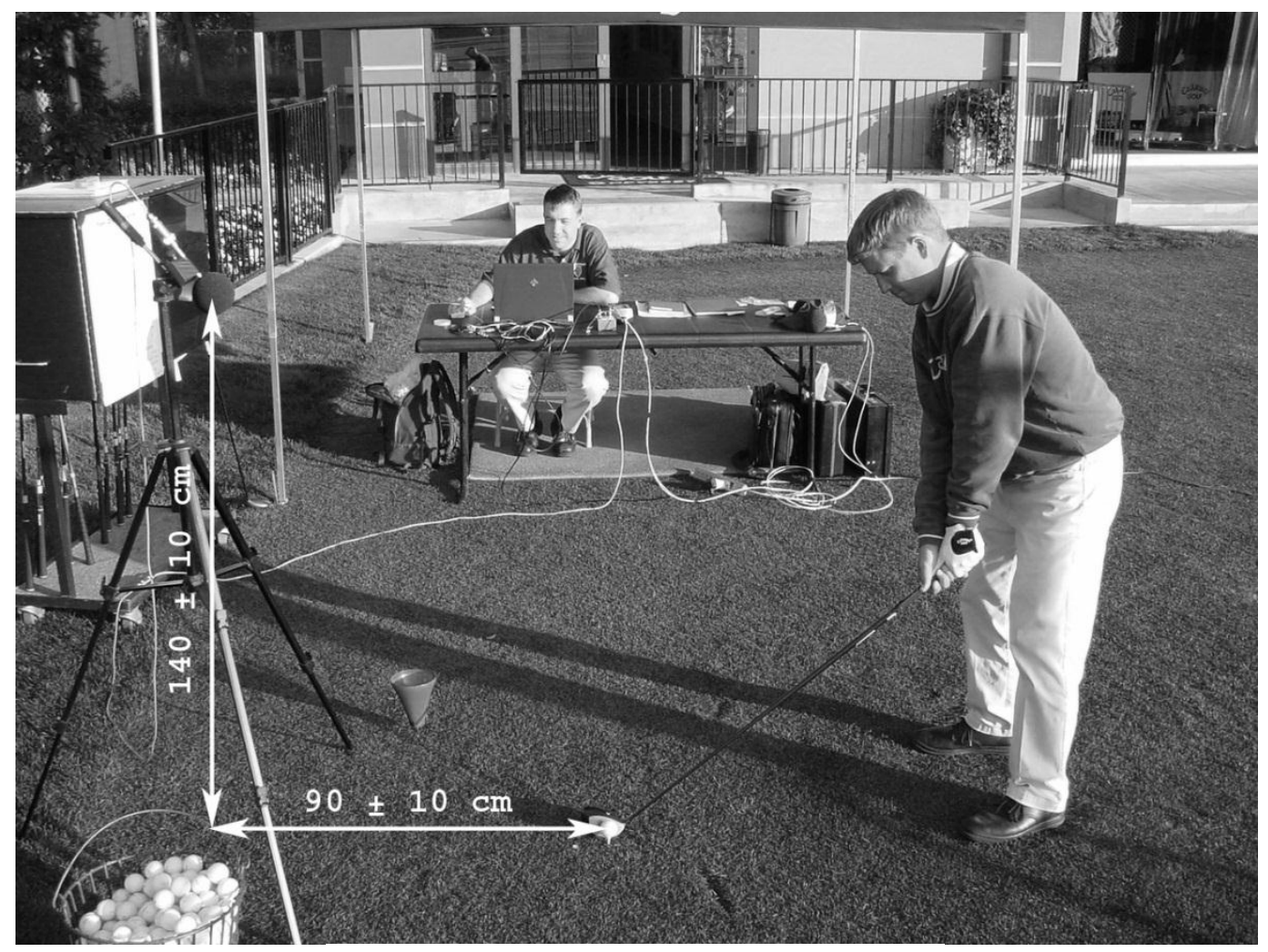

Figure 1. Microphone location in the test set-up

British males.

In order to cover the full range of impact sounds associated with different club designs, ten clubs were selected. Included were five modern titanium clubheads with varying sound characteristics, along with five older clubs with heads made of steel, laminated wood, persimmon and graphite. Nominally identical three-piece wound balls were used for every test.

All seventeen tests were conducted outdoors at driving ranges, where the golfers hit into a net to prevent the flight of the ball influencing their responses. On arrival, the test procedure was outlined to each participant. The five questions to be answered after each shot were described and also presented on a wallboard to ease recollection of the questions. The golfers were asked to avoid allowing preconceptions of clubs or manufacturers' equipment to affect their judgement and to rate each shot on its own merit.

Club order was randomised throughout the complete set of tests, according to a Latin square. Thirteen of the golfers hit five shots with each of the ten clubs; four of the golfers hit three shots with each club. All subjective and objective measurements were recorded for each shot played.

\section{RESULTS}

\subsection{CORRELATION WITHIN SUBJECTIVE DATA}

The five subjective ratings 'pleasantness', 'sharpness', 'loudness', 'hardness' and 'liveliness' were initially correlated with each other to investigate relationships between the feel characteristics. The Pearson method was used to measure the linear relationship between each combination of subjective ratings and, initially, correlation coefficients were calculated individually for each golfer to investigate whether any relationships that emerged held for all golfers.

Strong correlations were discovered between many of the feel characteristics, although considerable intrasubject variations were also observed. Based on the correlation coefficients, fourteen of the seventeen golfers tested in this study could be grouped into three distinct categories; the remaining three showed significant individual traits. For each of the three groups, the mean and standard deviation of the correlation coefficients for each pair of subjective ratings were calculated and the results are illustrated in Figure 2. Each line represents the relationship between the two feel characteristics it connects, with the mean and the standard deviation of the correlation coefficients for that group of golfers highlighted in bold and in italics respectively. Solid lines represent a positive correlation, whilst dashed lines represent a negative correlation. The individual correlation coefficients for each golfer are based on a sample size of approximately forty-five shots, implying that coefficients greater in magnitude than approximately 0.3 are significant. Mean coefficients less than 0.3 are enclosed in brackets and coloured grey to indicate that, if a golfer followed the mean trend of the group, these correlations would not be significant. 

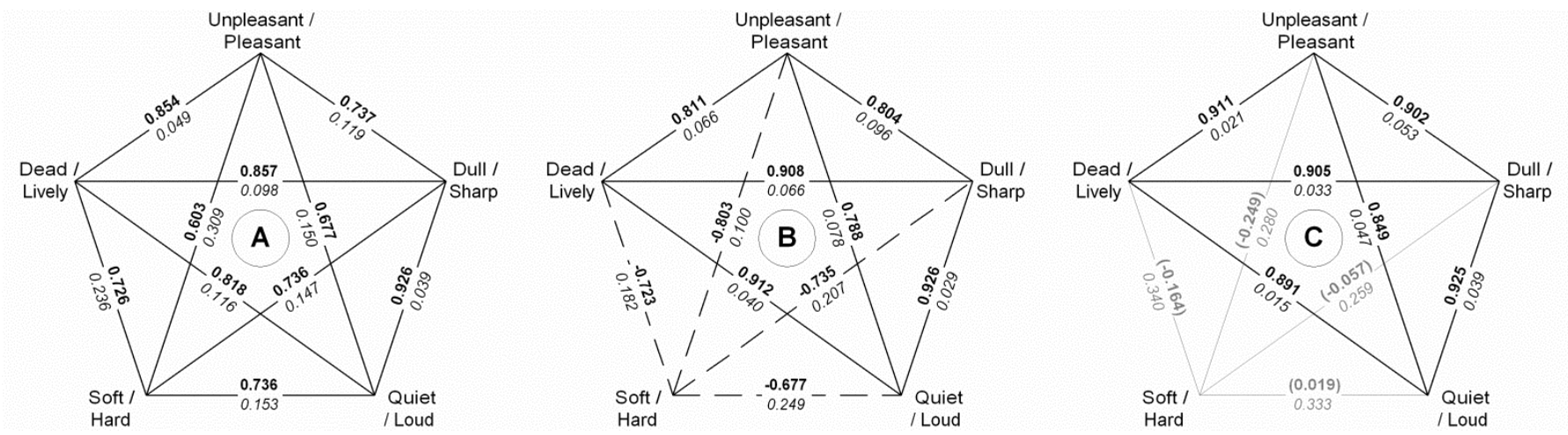

Figure 2. Mean Pearson correlation coefficients for each combination of feel characteristics for three different groups of golfers.

For the seven golfers in Group A, all of the feel characteristics are strongly related and the correlation coefficients are all positive, indicating that a pleasant feeling shot has a sharp, loud sound and a hard, lively feel. For the four subjects in Group B, the characteristics are also strongly related but the hardness of feel for this group of golfers is negatively related to the other characteristics, so a pleasant feeling shot also has a sharp, loud sound and a lively feel but has been rated by this group as having a soft feel. Three golfers formed the final set, Group C, and, again, the characteristics pleasantness and liveliness of feel and sharpness and loudness of sound are all strongly related in a positive manner. For this group of golfers, however, hardness of feel is unrelated to any of the other characteristics, with the majority of the coefficients being insignificant.

The only difference between the three groups of golfers in Figure 2 is the way in which the hardness of feel has been rated. For the golfers in Groups A and B, the hardness of feel is related to the impact sound, with the relationship differing between the two groups, whereas for golfers in Group C hardness of feel could be related to another form of feedback, such as tactile sensations. Alternatively, there may have been different interpretations of the question. A solution to this problem could not be determined from the available data, so the ratings of hardness of feel for all golfers were removed from the analysis, with an aim to resolve the issue during a future investigation.

Three golfers showed individual traits and did not fit into Groups A-C. In particular, for these three golfers, pleasantness of feel did not correlate strongly with either the perceived sharpness or loudness of the impact sound.
Again, it is not clear whether the weak correlations result from pleasantness being unrelated to the impact sound, whether they result from a different interpretation of the questions or alternatively whether they are an indication of unreliable subjects. Every golfer's opinion is of equal importance so, despite the trends for these three golfers being different from the majority, their data was retained.

To obtain an overall correlation coefficient for each combination of feel characteristics, all of the golfers' data needed to be combined but when scaled response questions are used participants typically use different ranges within the bounds of a scale. To overcome these variations, the responses were normalized [23] by taking each golfer's ratings for a particular characteristic, subtracting their mean rating and then dividing by their standard deviation. Thus, for each feel characteristic, the normalized ratings for each golfer had a mean of zero and a standard deviation of one.

Combining all the normalized data gave the correlation coefficients in Table 2, which are greater in magnitude than the coefficients obtained with the raw subjective data. There are strong correlations between the characteristics sharpness, loudness and liveliness of feel, with Pearson coefficients greater than 0.8 , indicating that almost all golfers agree that these characteristics are related in a positive manner, i.e. crisper, sharper sounds are perceived to be louder and are associated with a livelier feel. These three characteristics also correlate strongly and in a positive manner with the overall pleasantness of feel, but the Pearson coefficients are smaller, quantifying the extent of the variation between golfers discussed previously.

Table 2. Pearson correlation coefficients for each combination of normalized ratings ( $\rho$-value<o.oo1 for each coefficient)

\begin{tabular}{cccc}
\hline & $\begin{array}{c}\text { Unpleasant - } \\
\text { Pleasant Feel }\end{array}$ & $\begin{array}{c}\text { Dull - Sharp } \\
\text { Sound }\end{array}$ & $\begin{array}{c}\text { Quiet - Loud } \\
\text { Sound }\end{array}$ \\
\hline Dead - Lively Feel & 0.795 & 0.839 & 0.830 \\
Quiet - Loud Sound & 0.643 & 0.886 & \\
Dull - Sharp Sound & 0.705 & & \\
\hline
\end{tabular}




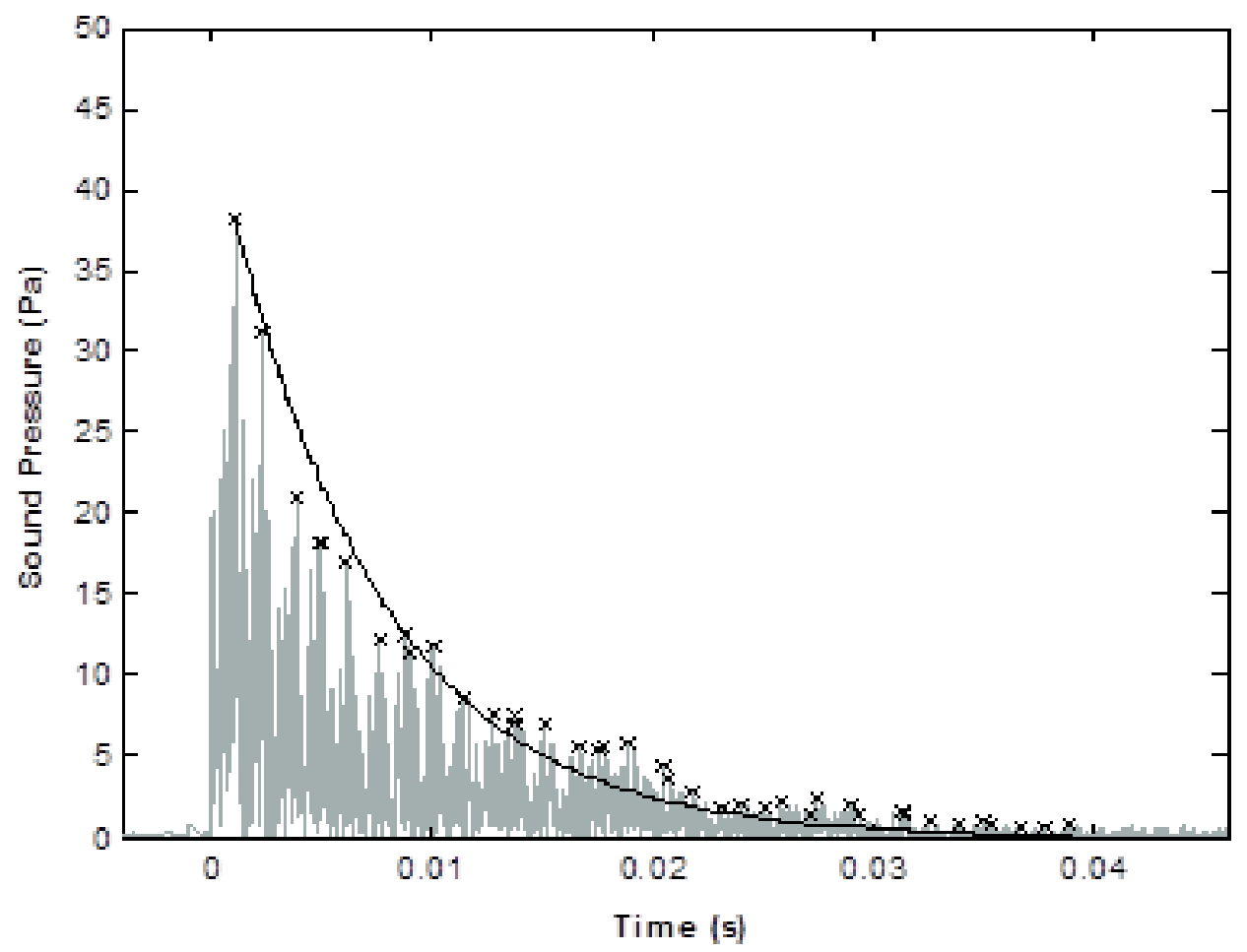

Figure 3. Fitting an exponential curve to the envelope of a sound measurement to calculate decay rate.

\subsection{OBJECTIVE DATA ANALYSIS}

In order to correlate the subjective and objective data, the following quantitative parameters were calculated for each impact sound measurement.

i) Decay rate - the value $b$ of an exponential curve $y(t)=a e^{b t}$ that follows the envelope of the sound measurement. As illustrated in Figure 3, the maximum absolute value of the measurement in each successive millisecond of data was identified (highlighted by a cross) and an exponential curve was fitted to these data points.

ii) Sound pressure level $(\mathrm{dB}(\mathrm{A})$ re $20 \mu \mathrm{Pa})$ calculated over the first $50 \mathrm{~ms}$ after impact to eliminate the sound of the ball hitting the catching net.

iii) Zwicker loudness (sone) - again based on the first 50 ms of data, calculated using standard procedures set out in ISO 532 [13].

iv) Centroid of a spectrum $(\mathrm{Hz})$ - the first moment a frequency spectrum, which has been shown to correlate well with the timbre of a sound [24]. v) Sharpness (acum) - calculated using Aures' method [21].

The centroid of a spectrum will differ if it is calculated from a magnitude or a power spectrum or if the logarithm of frequency is used; a logarithmically scaled frequency axis is often used in psychoacoustic studies of subjective sound quality. In this study, the centroid $f_{c}$, was calculated from the A-weighted amplitude, $a_{i}$, at each frequency, $f_{i}$, of a power spectrum containing $N$ data points using equation (1); all alternatives were calculated and this method gave the strongest correlation with the perceived sharpness of sound.

$$
f_{c}=10\left[\sum_{i=1}^{N} a_{i} \log \left(f_{i}\right) / \sum_{i=1}^{N} a_{i}\right]
$$

The sound pressure level and centroid of the spectrum were both based on A-weighted data. In fact, neither the

Table 3. Pearson correlation coefficients for each combination of sound parameters ( $\rho$-value<o.oor for each coefficient)

\begin{tabular}{ccccc}
\hline & Decay Rate & SPL(A) & $\begin{array}{c}\text { Zwicker } \\
\text { Loudness }\end{array}$ & $\begin{array}{c}\text { Aures } \\
\text { Sharpness }\end{array}$ \\
\hline Centroid of a spectrum & 0.762 & 0.792 & 0.675 & 0.839 \\
Aures Sharpness & 0.847 & 0.988 & 0.952 & \\
Zwicker Loudness & 0.794 & 0.975 & & \\
SPL(A) & 0.847 & & & \\
\hline
\end{tabular}


A or $C$ weightings influenced the strength of the correlations because, for a typical golf impact sound, the majority of content is in the region 1 to $10 \mathrm{kHz}$ and in this frequency range the weightings have little effect. Nonetheless, the A weighting was selected because it is the most commonly used weighting and it provides some immunity to low frequency noise typically encountered outdoors such as wind noise.

To investigate relationships between the sound characteristics of the clubs used, Pearson coefficients were calculated for each combination of objective parameters, as shown in Table 3. It can be seen from these results that all the sound parameters are strongly correlated, therefore the impact sounds with this set of clubs are generally either loud, sharp and long in duration or vice versa. This is because, compared to the traditional wooden clubs, the modern, hollow metal heads have low damping, higher natural frequencies and generate greater sound pressures.

\subsection{CORRELATION BETWEEN SUBJECTIVE AND OBJECTIVE DATA}

The objective data and the normalized subjective responses from all of the golfers were combined and Pearson correlation coefficients were calculated for each combination of sound parameter and subjective rating; the coefficients are given in Table 4. With 675 pairs of data contributing to each correlation, a Pearson coefficient with a magnitude of approximately 0.08 is significant at the 0.05 level.

It can be seen from these coefficients that the four feel characteristics all correlate in a positive manner with the sound parameters. The strongest correlations are with perceived loudness and sharpness of the impact sounds and also with liveliness of feel. The less strong correlations with pleasantness of feel are indicative of the differences of opinion between players, especially the three golfers not in Groups A-C, whose pleasantness ratings did not correlate significantly with the objective sound parameters either. It is possible that, for these golfers, the overall pleasantness of feel is not related to the sound but another form of feedback, such as tactile sensations. The strong correlations between ratings of liveliness of feel and the sound parameters supports the theory that emerged from the analysis of the subjective ratings that liveliness is related to the impact sound for the majority of golfers.

The golfers' perceptions of loudness correlate particularly strongly with SPL(A) and Zwicker loudness, which suggests that all the golfers used were sensitive to differences in impact sound and were able to report their perceptions consistently and reliably over the duration of the test. It is interesting to note that decay rate also correlates strongly with perceived loudness. This may be because all the longer duration sounds had higher sound pressure levels, as highlighted by Table 3, or alternatively because below $100 \mathrm{~ms}$ sound duration has a direct effect on loudness perception. Calculated sharpness correlates better with perceived sharpness than the centroid of a spectrum, which may be expected because the calculation procedures developed by Aures and von Bismark are based on psychophysical studies.

Perceived loudness has also correlated strongly with calculated sharpness $(r=0.829)$ indicating that sounds dominated by higher frequency components were perceived to be louder. In addition, perceived sharpness has correlated strongly with Zwicker loudness $(r=0.746)$ suggesting that the impact sounds were perceived to be sharper when the acoustic pressures were higher. Again, this may be because of the strong correlation between the Zwicker loudness and Aures sharpness levels of the impact sounds from the clubs used in this study. Alternatively, there may be an interaction between the perceived loudness and sharpness of a golf impact sound. Sharpness of continuous sounds, however, has only been found to increase by a factor of two for a level increment from 30 to $90 \mathrm{~dB}$ so its dependence on level is generally ignored when differences in sound pressure level are not large [12].

To illustrate the correlations, two combinations of data, Zwicker and perceived loudness and Aures and perceived sharpness, are plotted in two different styles in Figures 4 and 5 respectively. Figures $4 \mathrm{a}$ and $5 \mathrm{a}$ show, for each combination, a linear line of best fit through the individual data points. To obtain the graphs in Figures $4 \mathrm{~b}$ and $5 \mathrm{~b}$, the normalized ratings between -2 and +2 were divided into eight bands of equal width and the mean and standard deviation of the sound parameters were calculated for the data in each band.

Table 4. Pearson coefficients for each combination of sound parameter and feel characteristic ( $\rho$-value $<0.001$ for each coefficient)

\begin{tabular}{cccccc}
\hline Normalized & \multicolumn{5}{c}{ Sound Parameter } \\
Subjective Rating & $\begin{array}{c}\text { Decay } \\
\text { Rate }\end{array}$ & SPL(A) & $\begin{array}{c}\text { Zwicker } \\
\text { Loudness }\end{array}$ & $\begin{array}{c}\text { Centroid of } \\
\text { a spectrum }\end{array}$ & $\begin{array}{c}\text { Aures } \\
\text { Sharpness }\end{array}$ \\
\hline Unpleasant - pleasant feel & 0.440 & 0.514 & 0.503 & 0.432 & 0.508 \\
Dull - sharp sound & 0.704 & 0.775 & 0.746 & 0.664 & 0.769 \\
Quiet - loud sound & 0.764 & 0.836 & 0.815 & 0.694 & 0.829 \\
Dead - lively feel & 0.656 & 0.724 & 0.707 & 0.606 & 0.718 \\
\hline
\end{tabular}


a) Loudness of each impact sound as a function of the rating given

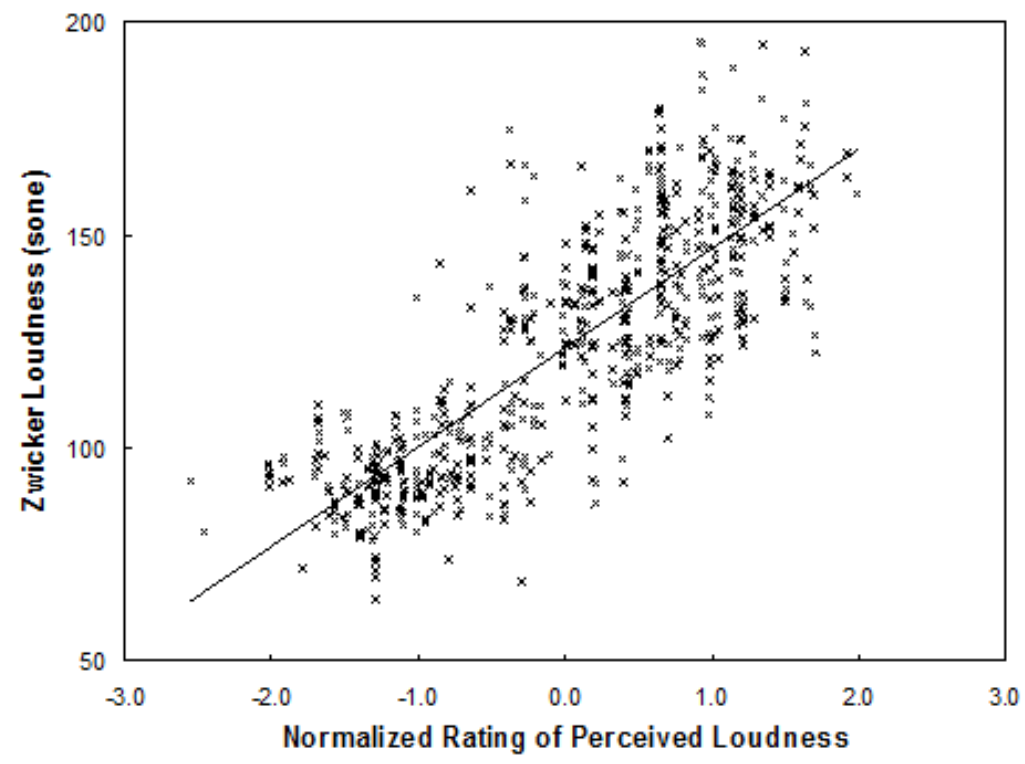

b) Mean loudness \pm one standard deviation of impact sounds in each band of ratings

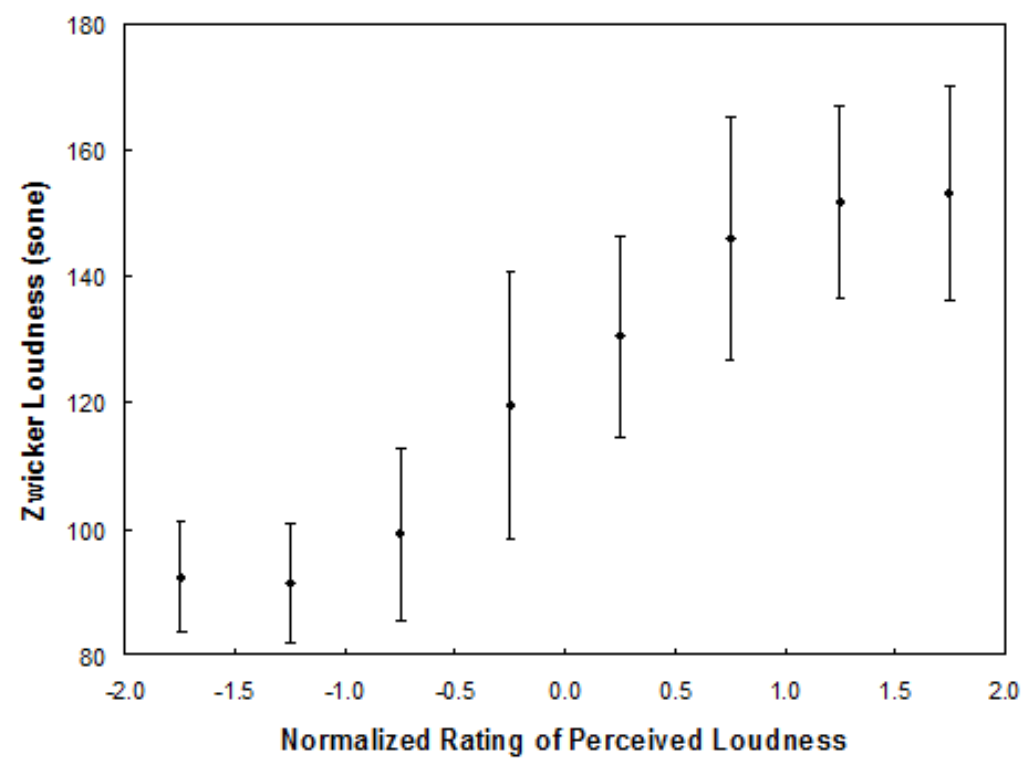

Figure 4. Correlation between perceived loudness and Zwicker loudness.

In Figure 4a, there is a strong positive trend through the data and it is clear why a large correlation coefficient of 0.815 was obtained. This conclusion is also supported by Figure $4 \mathrm{~b}$ but this representation of the data also enables another trend to be identified. The data only appears to have a positive linear relationship between the normalized ratings of -1 and +1 , outside of this region there is no relationship. For each band of ratings from 1 to 2, the mean Zwicker loudness is between 140 and 160 sone, whilst for each band of ratings from -1 to -2 the mean is between 80 and 100 sone. It is also apparent from this figure that Zwicker loudness had to differ by at least 30 sone for golfers to distinguish a difference.
The strong positive correlation $(r=0.796)$ between Aures and perceived sharpness is evident when Figure 5a is inspected. In this figure, there are more outlying data points, particularly the points above 10 acum with negative normalized ratings. These data points are likely to have resulted from off-centre shots, where a disproportionately low rating was given because the golfer was annoyed at hitting a poor shot. In Figure 5b, however, there only appears to be a positive linear relationship between Aures and perceived sharpness between the normalized ratings of -1 and +1 . Again, towards each end of the ratings scale, the linear relationship ceases to exist and at these extremes, sounds with similar sharpness levels received normalized ratings 
a) Sharpness of each impact sound as a function of the rating given

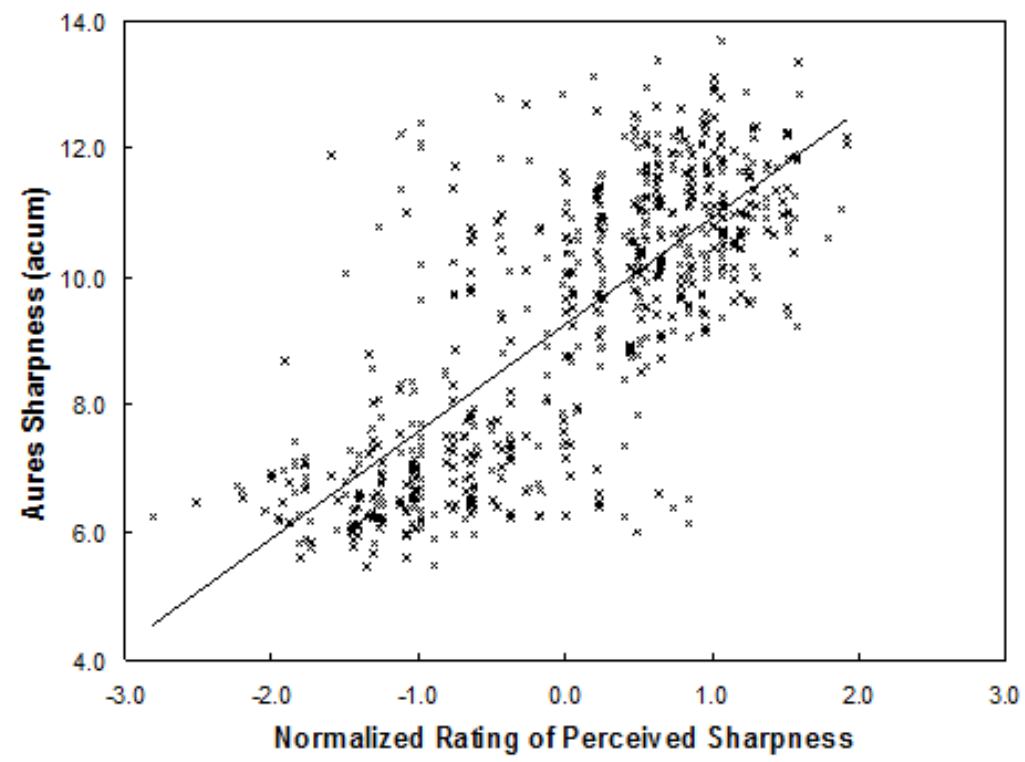

b) Mean sharpness \pm one standard deviation of impact sounds in each band of ratings

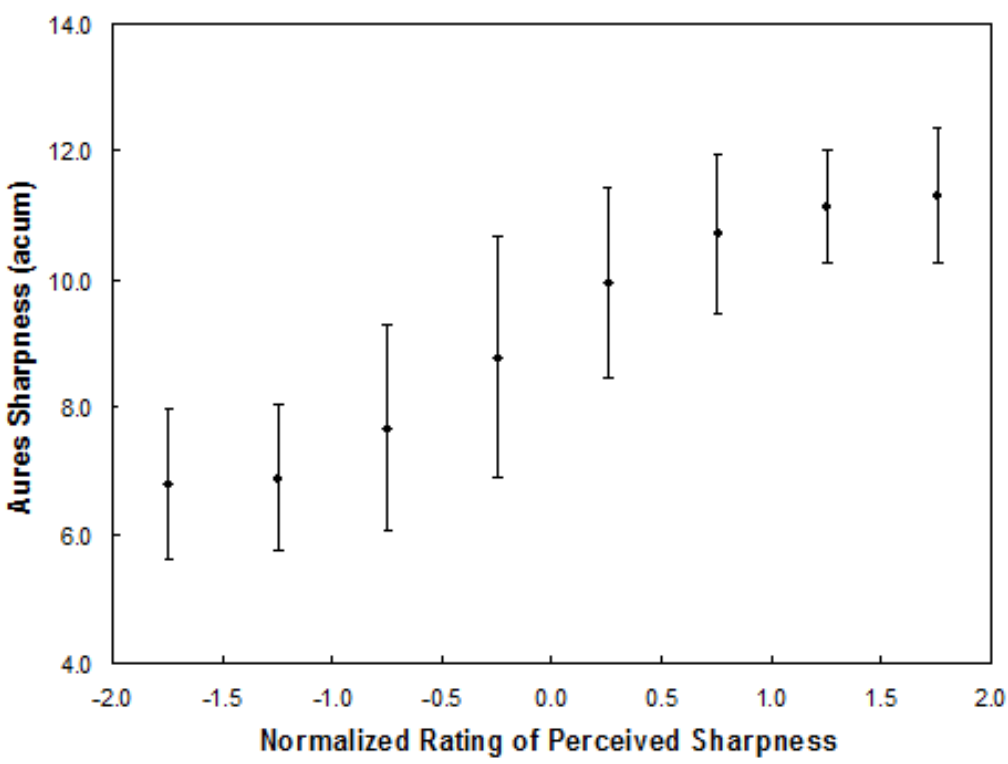

Figure 5. Correlation between perceived sharpness and Aures sharpness.

that differed by as much as 1.5 . Figure $5 \mathrm{~b}$ also suggests that a difference of at least 3 acum is required for the golfers to perceive a difference in the sharpness of the sounds. The trends in Figures $4 \mathrm{~b}$ and $5 \mathrm{~b}$ are likely to be because most of the data is contained within two clusters, depending on clubhead type. The loudness levels of the traditional wooden clubheads were typically between 80 and 100 sone but for the modern titanium clubheads, the levels were much greater, ranging from 120 to 180 sone. Similarly, the sharpness levels were much lower for the older style clubheads, ranging from 6 to 8 acum compared with 9 to 13 acum for the more recent clubs. The golfers were generally able to perceive the differences in loudness and sharpness between the two clubhead types but were less able to distinguish the more subtle variations in sound between similarly constructed heads. This may be a result of the test methodology chosen and, in particular, the number of clubs used and the sequential presentation of clubs. The golfers may have found it difficult to remember the impact sounds and the corresponding responses given to the clubs used earlier in the test when rating clubs later in the sequence. 


\section{DISCUSSION AND CONCLUSIONS}

The aim of this study was to develop test and analysis procedures suitable for investigating the relationship between the 'feel' of a golf shot and the impact sound. The players' perceptions of the pleasantness, hardness and liveliness of feel and the loudness and sharpness of the impact sounds were quantified using a questionnaire and correlated with parameters of the measured sound.

Analysis of the subjective ratings revealed some intersubject variability, particularly in the golfers' ratings of hardness of feel and as a result this characteristic was removed from the analysis. The remaining feel characteristics investigated correlated strongly and positively with each other; in general, a shot was rated as having a pleasant feel if it had, in the preferred vocabulary of the players, a loud, crisp, sharp sound and a lively feel. Normalizing the data to address inter-subject variations in the use of the scales improved the strength of these correlations.

Clubhead construction was found to have a major influence on impact sound characteristics. In this study, sound pressure levels varied from 85 to $110 \mathrm{~dB}(\mathrm{~A})$, loudness levels ranged from 80 to 180 sone and sharpness levels from 6 to 13 acum and the biggest differences were between the traditional and modern clubheads. The impact sounds from the solid wooden heads were shorter in duration and had loudness and sharpness levels towards the lower end of each range whilst the hollow titanium heads produced longer duration sounds that were both louder and sharper.

In general, the golfers were able to perceive the large differences in sound characteristics between the traditional and modern clubheads and this resulted in strong positive correlations between the subjective ratings and parameters of the objective data such as SPL(A), Zwicker loudness and Aures sharpness. For the golfers to be able to discriminate between sounds, however, the loudness levels needed to differ by at least 30 sone and sharpness by at least 3 acum. As a result, they were less able to distinguish the more subtle variations in sound characteristics between clubheads of a similar construction and the test methodology may have contributed to this. Neither was it possible, because of the nature of the subjective data, to analyse the repeatability and consistency of the subjects' responses. Alternative subjective data collection and analysis techniques could be considered for future tests, which may improve the likelihood of subtle differences emerging. For example, paired comparison tests place less demand on inexperienced test subjects, reduce the reliance on perceptual memory and responses can be tested for consistency.

It was hypothesized that the psychoacoustic metrics would correlate better with the subjective data than the more traditional metrics and, indeed, perceived sharpness did correlate better with Aures sharpness than with the centroid of a spectrum. Perceived loudness, however, correlated similarly with both SPL(A) and Zwicker loudness but then neither of these metrics fully reflects the perceived loudness of impulsive, impact sounds. For future tests, it may be necessary, therefore to consider the modification to Zwicker's loudness model for temporally variable sounds [25].

The golfers' sharpness ratings also correlated strongly with the loudness metrics, as did their loudness ratings with Aures sharpness. This suggests that the golfers' perceptions of sharpness may not only be influenced by frequency content but also by loudness and duration and perceptions of loudness by the frequencies and duration of the sound, in addition to sound pressure level. The strength of some of these correlations, however, will have been influenced by the clubs used in this study, which generally produced a loud, sharp, long duration sound or vice versa. In future, test clubs need to be incorporated for which frequency, duration and sound pressure level are less well correlated so that the effects of each sound parameter on perceived loudness and sharpness can be more conclusively determined.

This study is, to the authors' knowledge, the most extensive yet into the sound of a golf impact and its influence on players' perceptions. It has been found that golfers are able to distinguish differences in impact sounds between clubs and that sound characteristics are strongly correlated to the subjective perceptions of a golf club. This initial data analysis has already given specific insight into the meaning of feel descriptors such as 'lively' or 'dead' that form the typical vocabulary of the players. The suggested improvements to the test and analysis procedures that have emerged from this study will be an effective basis for any future tests.

\section{Acknowledgements}

The authors would like to thank the golfers for participating in the testing; without their donation of time and effort, this study could not have been conducted.

\section{References}

1. J. Roberts, R. Jones, C. Harwood, S. Mitchell and S. Rothberg, Human Perceptions of Sports Equipment under Playing Conditions. Journal of Sports Sciences 19 (7) (2001) pp.485-497.

2. A. L. Wicks, C. E. Knight, J. Neighbors and T. Blough, The Comparison of Forged Heads and Cast Heads for Golf Clubs, in: Proceedings of the 16th International Modal Analysis Conference Vol. 2, Santa Barbara, CA, USA, 1998, pp.1797-1802.

3. A. Hocknell, R. Jones and S. J. Rothberg, Engineering 'feel' in the design of golf clubs, in: S. J. Haake (Ed.), The Engineering of Sport: Proceedings of the 1st International Conference on the Engineering of Sport, A.A.Balkema, Rotterdam, NL, 1996.

4. A. Hocknell, S. R. Mitchell, R. Jones and S. J. Rothberg, Hollow golf club head modal characteristics: Determination and impact applications. Experimental Mechanics 38 (2) (1998) pp.140-146.

5. S. Kuwano, S. Namba, K. Ohta, H. Takeda, K. Sato and Y. Tsuji, Relation between envelope pattern and sound quality of impulsive sounds. Journal of the Acoustical Society of Japan (E) 20 (2) (1999) pp.153-155. 
6. J. R. Roberts, R. Jones, N. J. Mansfield and S. J. Rothberg, Evaluation of vibrotactile sensations in the 'feel' of a golf shot. Journal of Sound and Vibration (In press).

7. M. Hedrick and M. Twigg, The Feel Of A Golf Shot: Can We Measure It?, in: A. Cochran (Ed.), Golf: The Scientific Way, Aston Publishing Group, Hemel Hempstead, UK, 1994, pp.131-133.

8. M. Hedrick, Estimation of damping in graphite composite and steel golf club shafts, in: Proceedings of the 1995 SEM Spring Conference on Experimental Mechanics, Society for Experimental Mechanics, Michigan, USA, 1995.

9. L. Noble and H. Walker, Baseball Bat Inertial and Vibrational Characteristics and Discomfort Following Ball-Bat Impacts. Journal of Applied Biomechanics 10 (1994) pp.132-144.

10. C. L. Stroede, L. Noble and H. S. Walker, The effect of tennis racket string vibration dampers on racket handle vibrations and discomfort following impacts. Journal of Sports Sciences 17 (1999) pp.379-385.

11. J. R. Roberts, R. Jones and S. J. Rothberg, Measurement of contact time in short duration sports ball impacts: an experimental method and correlation with the perceptions of elite golfers. Sports Engineering 4 (4) (2001) pp.191-203.

12. E. Zwicker and H. Fastl, Psychoacoustics: Facts and Models, Springer, Berlin, 1999, 2nd edition.

13. International Organization for Standardization ISO 532, Acoustics - Methods for calculating loudness level, 1975.

14. F. Fahy and J. Walker, Fundamentals of Noise and Vibration, E \& FN Spon, London, 1998.

15. H. Fastl, The Psychoacoustics of Sound-Quality Evaluation. Acustica 83 (1997) pp.754-764.

16. J. R. Hassall and K. Zaveri, Acoustic Noise Measurements, Brüel and Kjær, Denmark, 1979, 4th edition.

17. W. L. Gulick, G. A. Gescheider and R. D. Frisina, Hearing: Physiological Acoustics, Neural Coding and Psychoacoustics, Oxford University Press, New York, 1989.

18. S. Coren, L. M. Ward and J. T. Enns, Sensation and Perception, Harcourt Brace College Publishers, Fort Worth, USA, 1999, 5 th edition.

19. J. M. Doughty and W. R. Garner, Pitch characteristics of short tones. I. Two kinds of pitch threshold. Journal of Experimental Psychology 37 (1947) pp.351-365.

20. G. von Bismark, Sharpness as an attribute of the timbre of steady sounds. Acustica 30 (1974) pp.159-172.

21. W. Aures, Berechnungsverfahren für sensorischen wohlklang beliebiger schallsignale (A procedure for calculating sensory pleasantness of various sounds). Acustica 59 (1985) pp.130-141.

22. R. C. Merkel and T. Blough, Dynamic Characterization and Comparison of Golf Clubs, in: Proceedings of the 17th International Modal Analysis Conference, FL, USA, 1999, pp.513517 .

23. N. J. Mansfield, P. Holmlund and R. Lundström, Comparison of subjective responses to vibration and shock with standard analysis methods and absorbed power. Journal of Sound and Vibration 230 (3) (200o) pp.477-491.

24. S. McAdams, J. W. Beauchamp and S. Meneguzzi, Discrimination of musical instrument sounds resynthesized with simplified spectrotemporal parameters. Journal of the Acoustical Society of America 105 (2) (1999) pp.882-897.

25. E. Zwicker, Procedure for calculating loudness of temporally variable sounds. Journal of the Acoustical Society of America 62 (3) (1977) pp.675-682. 